\title{
Los deportes adaptados como contribución a la educación en valores y a la mejora de las habilidades motrices: la opinión de los alumnos de Bachillerato \\ Adapted sports as a contribution to education in values and the improving motor skills: the opinion of high school students \\ *José Robles-Rodríguez, Manuel Tomás Abad-Robles, *Javier Giménez Fuentes-Guerra, **Pedro Benito-Peinado *Universidad de Huelva (España), **Universidad Politécnica de Madrid
}

Resumen: El objetivo de este estudio fue conocer la opinión del alumnado respecto a sus vivencias en las clases de educación física comparando los deportes tradicionales y los deportes adaptados en relación con el fomento de valores, la sensibilización social, la reflexión crítica y la participación, así como la habilidad motriz requerida para la práctica de los mismos. Con este fin, se encuestó a 103 estudiantes de Bachillerato del I.E.S. Diego Angulo, ubicado en la población de Valverde del Camino (Huelva), con una edad media de $17.6 \pm 0.65$. Los resultados mostraron que los deportes adaptados fomentaban en mayor medida la igualdad de oportunidades (tradicionales $=2.19 \pm 1.04$; adaptados $=3.85 \pm 0.40 ; p<0.001$ ), el respeto (tradicionales $=2.84 \pm 0.87$; adaptados $=3.56 \pm 0.65 ; p<0.001$ ) y la sensibilización social (tradicionales $=1.84 \pm 1.08$; adaptados $=3.80 \pm 0.58 ; p<0.001$ ). Se concluye proponiendo la incorporación de los deportes adaptados al currículum de educación física en Secundaria.

Palabras clave: Currículum, Educación Física, Educación inclusiva.

Abstract: The aim of this study was to know the opinion of students regarding their experiences in physical education classes by comparing the traditional and adapted sports in relation to the promotion of values, social awareness, critical thinking and participation as well as the driving skills required for the practice of the same. To this end, 103 undergraduate students were surveyed in I.E.S. Diego Angulo from Valverde del Camino, with a middle age of $17.6 \pm 0.65$. Results showed that the adapted sports encouraged greater equality of opportunity (traditional $2.19 \pm 1.04$; adapted $3.85 \pm$ 0.40 ; p <0.001), respect (traditional $2.84 \pm 0.87$, adapted $3.56 \pm 0.65 ; \mathrm{p}<0.001$ ) and social awareness (traditional $1.84 \pm 1.08$; adapted $3.80 \pm 0.58$; $\mathrm{p}<0.001$ ). It concludes by proposing the incorporation of sports adapted to the curriculum of physical education in high school.

Keywords: Curriculum, Physical education, Inclusive education.

\section{Introducción}

La importancia de una adecuada educación inclusiva en el ámbito escolar demanda, cada día más, una mayor concienciación por parte de las personas sin discapacidad. Uno de los factores clave para el éxito de la inclusión es un contexto social favorable, el cual incluya una actitud positiva por parte de profesores, alumnado y padres (Reina, López, Jimenez, García-Calvo y Hutzler, 2011). De esta forma, los profesores deben ayudar a sus estudiantes a ser conscientes y respetuosos con las diferencias individuales, incluyendo las discapacidades(Columna, Arndt, Lieberman y Yang, 2009). Cabe destacar que, en el ámbito de la educación física y el deporte, la integración se refiere a las adaptaciones que se llevan a cabo en las tareas con el fin de que las personas con discapacidad puedan tomar parte en la actividad de una forma lo más normalizada posible, mientras que en la inclusión las actividades son planteadas de manera que las personas con discapacidad tengan las mismas oportunidades de participación y práctica que el resto de participantes (Sanz y Reina, 2012).

Por otro lado, los deportes de equipo conforman el contenido más utilizado por los docentes en el currículum de educación física, (Robles, 2009a; Ureña, Alarcón y Ureña, 2009), además de ser el aspecto más demandado por el alumnado (Hill y Cleven, 2006). Sin embargo, la práctica de los mismos no parece facilitar la inclusión de los alumnos con discapacidad, ya que no permiten una plena participación de éstos, debido, fundamentalmente, a las exigencias físicas, intensidad, control y duración de las habilidades requeridas (Smith, 2004). Por otro lado, la introducción en la enseñanza de la educación física de los deportes adaptados se considera un medio apropiado para promover la inclusión (Ocete, Pérez-Tejero y Coterón, 2014) y establecer un currículum de la asignatura más diferenciado y comprensivo (Davis, Rocco-Dillon, Grenier, Martínez, y Aenchbacker, 2012). En este sentido, Connel (1997) aporta la necesidad de un currículum contrahegemónico que favorezca la justicia curricular, de manera que se materialicen los intereses y las perspectivas de los menos favorecidos. Estos deportes pueden proporcionar numerosas oportunidades para incrementar la concienciación y la apreciación de los estudiantes hacia las personas con discapacidad (Vaughn, 2012), ya que el proceso inclusivo requiere de

Fecha recepción: 06-04-16. Fecha de aceptación: 15-08-16

José Robles-Rodríguez

jose.robles@dempc.uhu.es una reestructuración de las percepciones intragrupo e intergrupo(Segura, Martínez-Ferrer, Guerra y Barnet, 2013). Además, la inclusión de deportes adaptados en los programas de educación física puede beneficiar a todos los estudiantes, tanto desde el punto de vista de la actividad física como de la integración social (Grenier y Kearns, 2012). No obstante, existe un gran desconocimiento respecto a este tipo de deportes $\mathrm{y}$, normalmente, las personas sin discapacidad no son conscientes de los efectos positivos que puede tener la práctica deportiva incluyendo a compañeros con discapacidad (Eminovic, Nikic, Stojkovic, y Pacic, 2009), lo cual subraya la importancia de incluir este tipo de deportes en el currículum de la educación física, ya que éstos son accesibles y beneficiosos para todos los estudiantes (Grenier y Kearns, 2012).

La puesta en práctica de los deportes adaptados a través de planteamientos metodológicos adecuados a las características del contexto en el que nos situamos, puede ayudar a paliar la falta de actitud crítica y reflexiva hacia prácticas deportivas que sólo satisfacen a los más capacitados físicamente, y la crisis generalizada de valores personales y sociales (Andréu, 2006). Spencer-Cavaliere y Peers (2011) plantean la integración inversa, utilizada anteriormente por Brasile (1990) como medio para favorecer la inclusión de estudiantes con discapacidad. La integración inversa consiste en que personas sin discapacidad participan en deportes creados específicamente para personas discapacitadas (deportes adaptados). Los deportes adaptados, pues, se convierten en un verdadero agente integrador (Brasile, 1990).

Teniendo en cuenta las características del contexto en el que se sitúa el estudio, es decir, un grupo-clase en el que no había sujetos con discapacidad física, la intención del trabajo se centró fundamentalmente en valorar el deporte adaptado como herramienta de sensibilización hacia las personas con discapacidad, no sin menospreciar que dicho contenido puede ser utilizado como un instrumento de inclusión muy válido cuando tengamos alumnos con discapacidad en el grupo-clase.

De esta forma, el objetivo de este estudio fue conocer la opinión del alumnado respecto a sus vivencias en las clases de educación física comparando los deportes tradicionales y los deportes adaptados en relación con el fomento de valores, la sensibilización social, la reflexión crítica y la participación, así como la habilidad motriz requerida para la práctica de los mismos.

\section{Material y método}

\section{Participantes}

La investigación se centra en el alumnado de Bachillerato (edad 
media de 17.6 \pm 0.65 ) del I.E.S. Diego Angulo, ubicado en la población de Valverde del Camino (Huelva). En el estudio han participado 103 estudiantes, de los cuales el $42.7 \%$ ( $n=44)$ eran hombres y el $57.3 \%$ $(\mathrm{n}=59)$ mujeres, todos sin discapacidad. El 56.8\% de los chicos y el 18.7 $\%$ de las chicas realizaban actividad físico-deportiva de manera continuada. Además, el 31.8\% de los chicos y el 55.9\% de las chicas la llevaban a cabo de manera esporádica, mientras que el $11.4 \%$ de los chicos y el 25.4\% de las chicas no hacían, en el momento de la encuesta, actividad físico-deportiva.

\section{Procedimiento}

Durante el curso académico 2012/13 se estableció contacto con diferentes centros educativos con el fin de conocer los recursos de instalaciones y de materiales deportivos con los que contaban. Finalmente se optó por un centro que cumplía con los requisitos que aseguraba un buen desarrollo de todos los contenidos deportivos establecidos para el estudio a realizar. Durante ese curso se diseñaron las distintas unidades didácticas (UU.DD.) siguiendo una metodología global de la enseñanza del deporte, caracterizada fundamentalmente por desarrollar en los alumnos/as una conciencia táctica y favoreciendo la toma de decisiones (Bunker y Thorpe, 1982; Mitchel, Oslin y Griffin, 1995, 2006; Turner y Martinek, 1995). Dichas UU.DD. fueron impartidas por el profesor de educación física del centro educativo, quien no pertenecía al equipo investigador. Con el fin de asegurar la correcta intervención pedagógica, el docente encargado de impartir dichas UU.DD. participó en sesiones de trabajo dentro de un grupo de discusión desarrollado por los responsables del estudio, donde se trabajaba en torno a modelo de enseñanza comprensivo. En dicho grupo de discusión se elaboraron las distintas UU.DD. con el fin de asegurar que las unidades cumplían los aspectos fundamentales para el desarrollo de la investigación.

Durante el primer trimestre del curso escolar 2013/14 se pusieron en práctica tres UU.DD. dedicadas a deportes más tradicionales, concretamente, baloncesto, balonmano y voleibol. Cada unidad didáctica estuvo compuesta de 8 sesiones y, una vez finalizadas las mismas se implementó una pequeña competición (participativa y nada selectiva ni discriminatoria) donde se pusieron en práctica los distintos deportes. Todos los equipos eran mixtos. Finalmente, se pasó un cuestionario donde el alumnado debía responder atendiendo a lo vivenciado durante las clases dedicadas a los deportes tradicionales.

A lo largo del segundo trimestre se implementaron otras tres UU.DD. de deportes adaptados. En éstas se trabajó voleibol sentado, bádminton sentado y goalball. Cada U.D. estaba compuesta por 8 sesiones. Igualmente, al finalizar se hizo un sencillo campeonato (con las mismas características que el anterior) donde se jugó a los tres deportes y donde los equipos fueron mixtos. Una vez terminado el campeonato se pasó al alumnado el mismo cuestionario con la salvedad de que ahora debían responder en relación con lo experimentado en los deportes adaptados.

\section{Instrumento}

El instrumento utilizado en este trabajo fue un cuestionario elaborado por el propio equipo de investigación, con el que se analizaron las siguientes dimensiones: valores, participación, sensibilización social, reflexión crítica y habilidades y destrezas motrices. Se elaboraron las preguntas que conformarían cada una de las dimensiones, quedando compuesto por 30 ítems, los cinco primeros referidos a datos sociodemográficos. El cuestionario estuvo formado por preguntas actitudinales en las que se utilizó una escala Likert que fue de 1 (poco de acuerdo) a 4 (muy de acuerdo). El instrumento fue revisado y validado por expertos en el diseño y confección de cuestionarios. De esta manera, la validez de contenido se llevó a cabo siguiendo las sugerencias de Buendía (1992) y Díaz (2005). Una vez elaborado el cuestionario, se realizó un estudio piloto aplicándose el instrumento a una muestra incidental de 15 estudiantes de $2^{\circ}$ de Bachillerato con el fin de corregir posibles problemas y dificultades que pudiese presentar el cuestionario, no utilizándose los datos hallados en el presente estudio.

\section{Análisis estadístico}

Para comprobar la fiabilidad y consistencia interna del cuestionario, se aplicó la prueba estadística Alpha de Cronbach, obteniendo los siguientes valores: 0.856 (deportes tradicionales) y 0.746 (deportes adaptados). Atendiendo al Alpha por dimensiones en el cuestionario referido a deportes tradicionales obtenemos los siguientes datos: valores 0,738 ; participación 0,715; sensibilización social 0,768; reflexión crítica 0,507 y habilidades y destrezas motrices 0,628. Respecto al Alpha de las dimensiones del cuestionario referido a deportes adaptados obtenemos los siguientes datos: valores 0,783 ; participación; sensibilización social 0,618; reflexión crítica 0,572 y habilidades y destrezas motrices 0,556.

Se calcularon las medias y las desviaciones estándar de todas las respuestas recogidas en los cuestionarios. Se realizó una T de Student para muestras relacionadas para observar las diferencias entre deporte tradicional y deporte adaptado. Se llevó a cabo un análisis de la varianza (ANOVA) de medidas repetidas con factor intrasujeto (tradicional $\mathrm{x}$ adaptado) y con factor intersujeto (dimensión y sexo) con el fin de observar las interacciones entre los factores analizados. Para las comparaciones por pares se utilizó el estadístico de Bonferroni como Post Hoc. La simetría y esfericidad fueron verificadas con el test de Mauchli. Cuando no se pudo asumir la esfericidad los valores de la F, éstos fueron corregidos con el procedimiento de Greenhouse-Geisser. Los análisis fueron realizados con el paquete estadístico SPSS v. 15.0 para Windows. Las diferencias de las medias fueron consideradas significativas cuando el valor de alfa fue menor a 0.05 .

\section{Resultados}

En la tabla 1 se muestran las diferencias entre las dimensiones estudiadas. El ANOVAmostró una interacción significativa triple entre los factores dimensión, sexo y tipo de deporte $(\mathrm{F}(1,1,5)=8.4$ con $\mathrm{p}<0.03)$.

En general existen diferencias entre deporte tradicional frente a adaptado en todas las dimensiones, excepto en la dimensión habilidades y destrezas motrices.

Dentro de deporte tradicional, las medias son superiores en mujeres con respecto a hombres en todas las dimensiones, excepto en habilidades y destrezas motrices. En el caso de deporte adaptado, no existen diferencias debidas al género.

Además, teniendo en cuenta el género, las respuestas son significativamente más altas en deporte adaptado para todas las dimensiones excepto para habilidades y destrezas motrices, tanto en hombres como mujeres.

\section{Dimensión «valores»}

En esta dimensión se observaron diferencias significativas en relación con el fomento de valores entre los deportes tradicionales y los deportes adaptados. En cuatro de los cinco ítems analizados en esta

Tabla 1.

Resultados del ANOVA para deportes tradicionales frente adaptados.

\begin{tabular}{|c|c|c|c|c|c|c|c|c|c|c|c|c|}
\hline \multirow[b]{3}{*}{ Dimensión } & \multicolumn{6}{|c|}{ Tradicional } & \multicolumn{6}{|c|}{ Adaptado } \\
\hline & \multicolumn{2}{|c|}{ Hombre } & \multicolumn{2}{|c|}{ Mujer } & \multicolumn{2}{|c|}{ Todos } & \multicolumn{2}{|c|}{ Hombre } & \multicolumn{2}{|c|}{ Mujer } & \multicolumn{2}{|c|}{ Todos } \\
\hline & Media & ETM & Media & ETM & Media & ETM & Media & ETM & Media & ETM & Media & ETM \\
\hline Valores & 2.35 & 0.14 & $2.94 *$ & 0.10 & 2.64 & 0.12 & $3.41^{*}$ & 0.12 & $3.50^{\#}$ & 0.09 & $3.46^{¥}$ & 0.10 \\
\hline Participación & 2.34 & 0.14 & $2.91 *$ & 0.10 & 2.62 & 0.12 & $3.47^{*}$ & 0.12 & $3.47 \#$ & 0.09 & $3.47^{*}$ & 0.10 \\
\hline Sensibilización social & 1.73 & 0.14 & $2.52 *$ & 0.10 & 2.13 & 0.12 & $3.59 *$ & 0.12 & $3.67^{\#}$ & 0.09 & $3.63^{\sharp}$ & 0.11 \\
\hline Reflexión crítica & 2.18 & 0.14 & $2.68^{* *}$ & 0.10 & 2.43 & 0.12 & $3.08^{*}$ & 0.12 & $3.05^{\#}$ & 0.09 & $3.06^{*}$ & 0.11 \\
\hline Habilidades y destrezas motrices & 2.34 & 0.14 & 2.47 & 0.10 & 2.41 & 0.12 & 2.32 & 0.12 & 2.39 & 0.09 & 2.35 & 0.11 \\
\hline
\end{tabular}


dimensión quedó constancia de un mayor fomento de valores educativos en la práctica de deportes adaptados frente a los deportes tradicionales. La diferencia fue mayor en favor de los deportes adaptados en el ítem referido a la igualdad de oportunidades. También se detectó una gran desigualdad en cuanto a favorecer el respeto durante la práctica deportiva a favor de los deportes adaptados. En tercer lugar, se encontraron diferencias, aunque algo menores, cuando se analizó el ítem relacionado con el desarrollo como persona. Respecto a favorecer las relaciones entre los alumnos, se halló una variación pequeña a favor de los deportes adaptados (tabla 2).

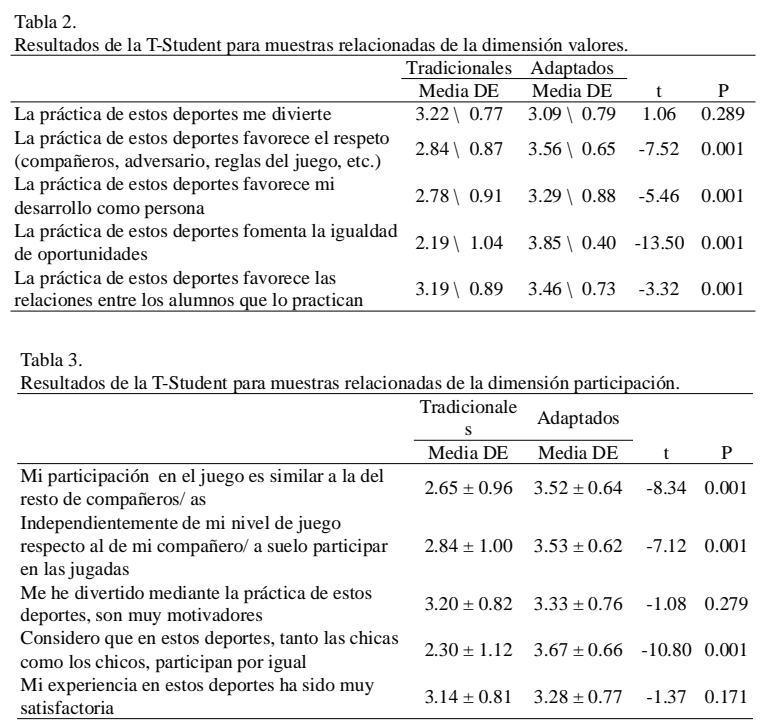

\section{Dimensión «participación»}

En esta dimensión relacionada con la participación del alumnado en las sesiones de educación física se hallaron diferencias significativas en favor de los deportes adaptados en los siguientes ítems: participación similar entre chicos y chicas; participación similar al resto de compañeros y compañeras; y participación en el juego independientemente de mi nivel de aptitud. No se encontraron diferencias significativas cuando analizamos los ítems relacionados con la motivación y en la satisfacción de una u otra práctica (tabla 3).

\section{Dimensión «sensibilización social»}

Los datos obtenidos en esta dimensión mostraron diferencias significativas entre los deportes tradicionales y los deportes adaptados. En este sentido, los resultados mostraron que los alumnos y alumnas consideraron que los deportes adaptados favorecían en mayor medida las

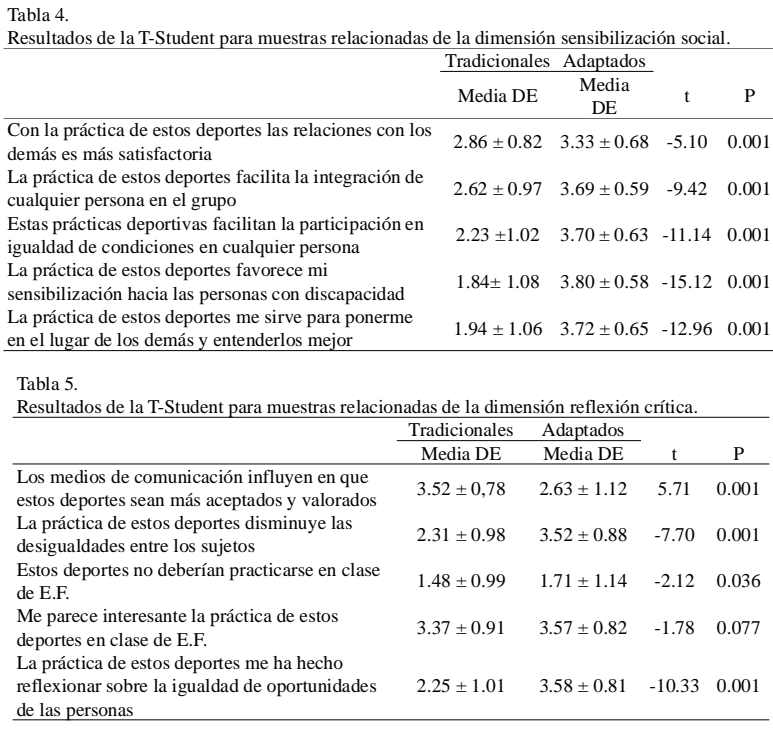

relaciones con los demás y la integración. Respecto al fomento de una participación en igualdad, los deportes adaptados volvieron a presentar puntuaciones más altas que los tradicionales. Según los encuestados, los deportes adaptados favorecieron, en mayor medida, la sensibilización hacia personas con discapacidad y el fomento de la empatía (tabla 4).

\section{Dimensión «reflexión crítica»}

En esta dimensión los datos presentaron diferencias significativas entre los deportes tradicionales y los deportes adaptados, excepto en el interés del alumnado hacia la presencia de estos deportes en las clases de educación física, aunque la diferencia es mínima. De esta forma, los resultados reflejaron que el alumnado consideró que los medios de comunicación influían en que los deportes tradicionales fuesen más aceptados y valorados que los deportes adaptados. En relación con la disminución de las desigualdades, se encontraron diferencias significativas a favor de los deportes adaptados. Por último, la práctica de deportes adaptados presentó valores más altos en cuanto al fomento de la reflexión sobre la igualdad de oportunidades de las personas (tabla 5).

\section{Dimensión «habilidadesy destrezas motrices»}

En relación con la pregunta «me resulta fácil jugar a estos deportes porque los gestos deportivos son fáciles de realizar», no se observaron diferencias significativas en ambos grupos de deportes, aunque sí es cierto que los deportes adaptados presentaron datos más favorables. Los datos tampoco mostraron diferencias significativas en relación con que la práctica de estos deportes de manera regular fuese a contribuir a que los alumnos se divirtieran más. Por otro lado, respecto al dominio de los gestos técnico-tácticos, se observaron diferencias significativas a favor de que para poder jugar era necesario dominar en mayor medida los gestos técnico-tácticos en los deportes tradicionales (tabla 6).

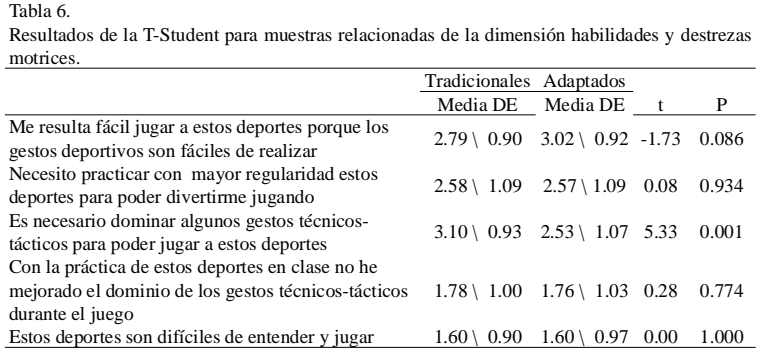

\section{Discusión}

La utilización de deportes adaptados en las clases de educación física frente a los deportes tradicionales, presenta beneficios a favor de los primeros en relación con el desarrollo de diferentes valores, lo cual coincide con lo hallado en otras investigaciones en cuanto a la igualdad de oportunidades (Monge y Monge, 2009), y a la mejora de las relaciones (Brasile, 1990; Seymour, Reid y Bloom, 2009). Además, Lavega, Planas y Ruiz (2014) obtuvieron resultados similares en su estudio sobre juegos cooperativos e inclusión en clases de educación física. Desde otra perspectiva, pero confirmando los resultados obtenidos, Smith (2004) incidió en que los deportes tradicionales no eran útiles para fomentar la igualdad en las clases de educación física, por lo que propone incorporar al currículum nuevos contenidos que nos ayuden en este sentido. También sería importante promover habilidades sociales (Saiz, Prieto, Gutiérrez y Gil, 2016) entre personas con y sin discapacidad, así como favorecer conductas apropiadas en las clases de educación física (Madrid, Prieto-Ayuso, Samalot-Rivera y Gil, 2016).

Los datos hallados revelan que los deportes adaptados fomentan en mayor medida la participación general del alumnado, independientemente de su nivel, en las sesiones de educación física, fundamentalmente de las chicas. Numerosas investigaciones demostraron que éstas participan menos en las clases deEducación Física(Chepyator-Thomson y Ennis, 1997; Martínez de Quel, Fernández y Camacho, 2010), y que la participación descendía más todavía si utilizamos actividades depor- 
tivas tradicionales en nuestras clases (González, 2005). Al respecto, Kyles y Lounsbery (2004) indicaron que eran necesarios nuevos planteamientos críticos, y más integrales y educativos que favorecieran la práctica de las adolescentes de Educación Secundaria. Todas estas ideas son refrendadas en nuestro estudio y también en otros como el de Robles (2009b), quien introdujo nuevos contenidos deportivos como el tchoukball para aumentar la motivación y la participación del alumnado en clase, especialmente entre las chicas.

Los resultados en la dimensión sensibilización social muestran que los encuestados manifestaban claramente que la práctica de deportes adaptados favorece, más que los deportes tradicionales, la sensibilización social hacia las personas con discapacidad. Liu, Kudlácek y Jesina (2010) y Santana y Garoz (2013) encontraron que la práctica de deportes adaptados incrementaba la actitud favorable de los estudiantes hacia la inclusión de personas discapacitadas. En este sentido, Columna et al. (2009) afirmaron que uno de los métodos para sensibilizar a los estudiantes sin discapacidad es tener experiencias similares a las que tienen las personas discapacitadas. Esta circunstancia tiene una gran importancia, ya que algunos estudios han demostrado que una de las barreras más comunes hacia la participación de las personas con discapacidad en el deporte es la existencia de actitudes negativas, indiferencia y prejuicios sociales hacia éstas (Eminovic et al., 2009; Molina y Valenciano, 2010; Savino, Rio, Gomez, 2015). Además, los datos expresan que las chicas muestran una actitud más favorable hacia la inclusión de los deportes adaptados en las clases de educación física que los chicos, lo cual coincide con lo encontrado por Hutzler (2003), Liu et al. (2010) y Reina et al. (2011). No obstante, Segura et al. (2013), en un contexto diferente, hallaron que los hombres (deportistas, técnicos y gestores de federaciones deportivas de deportes para personas con discapacidad) mostraban una actitud más positiva hacia la inclusión social que las mujeres.

En relación con la dimensión reflexión crítica, los encuestados consideran que los deportes adaptados promueven, en mayor medida que los deportes tradicionales, la reflexión crítica. Tal como mostraron Eminovic et al. (2009), muchas personas tienen un gran desconocimiento sobre los deportes adaptados, ya que no han tenido la oportunidad de verlos por televisión o leer sobre éstos en la prensa, ni están informados sobre la existencia de asociaciones o clubes deportivos para personas con discapacidad. En esta línea, la práctica de deportes adaptados puede favorecer tanto el conocimiento de los estudiantes sobre los mismos, como lo que significa tener una discapacidad, disminuyendo las desigualdades entre los sujetos y fomentando la reflexión sobre la igualdad de oportunidades.

La dimensión habilidades y destrezas motrices no muestra diferencias significativas entre ambos tipos de deportes. No obstante, los encuestados opinan que los deportes adaptados presentan gestos técnicos-tácticos más sencillos, por lo que les resultan más fáciles de dominar. Según Cockburn (2000), el nivel de habilidad influye directamente en la satisfacción de los participantes hacia la práctica de determinada actividad. Grenier y Kearns (2012) afirmaron que el atractivo de la aceptación de los deportes adaptados en la población en general durante las clases de educación física se debía a la facilidad en cuanto a la ejecución de los gestos deportivos.

En definitiva, los datos confirman una de las interesantes conclusiones del estudio de Gil-Madrona, Cachón-Zagalaz, Díaz-Suárez, ValdiviaMoral y Zagalaz-Sánchez (2014), en las que dejan claro que se precisa un nuevo modelo curricular de intervención educativa que sea efectivo y que tenga en cuenta las características particulares y niveles de habilidad de los estudiantes, lo cual también es destacado por Izquierdo-Gómez y Díaz-Cueto (en prensa). De esta manera, se estima de gran importancia la incorporación de los deportes adaptados al currículum de educación física en la E.S.O.

Uno de los factores limitantes de nuestro estudio fue la existencia de pocas investigaciones similares donde se comparen los deportes tradicionales y los adaptados en las clases de educación física en Secundaria. Otra de las dificultades encontradas estuvo relacionada con la aparición de ciertas reticencias iniciales a la hora de poner en práctica deportes adaptados, pudiendo ser debido al desconocimiento, estereotipos, creencias o prejuicios hacia la discapacidad. Por este motivo, y dada la importancia que tiene la incorporación de deportes adaptados al currículum de educación física, sería conveniente que futuros trabajos estudiaran el uso de éstos en las distintas etapas educativas, y en clases en las que haya alumnos con y sin discapacidad.

\section{Conclusiones}

La práctica de deportes adaptados dentro de las clases de educación física en bachillerato fomenta en mayor medida, en comparación con los deportes tradicionales, la promoción de valores educativos como la igualdad de oportunidades, el respeto, el desarrollo como persona, las relaciones con los demás y la integración.

La práctica de deportes adaptados en clase de educación física favorece más la participación de todo el alumnado, independientemente del género o del nivel de aptitud.

Los medios de comunicación influyen de forma clara en que los deportes tradicionales sean más aceptados y valorados, por parte de los estudiantes de Bachillerato, que los deportes adaptados.

\section{Referencias}

Andreu, E. (2006). El deporte y los valores humanos. Journal of human sport and exercise. 1, 7-14.

Brasile, F. M. (1990). Wheelchair sports: a new perspective on integration. Adapted Physical Quaterly, 7, 3-11.

Buendía, L. (1992). Técnicas e instrumentos de recogida de datos. En: Colás, M. P., Buendía, L. (Eds.), Investigación Educativa (pp. 201-248). Sevilla:Alfar, S.A.

Bunker, D. y Thorpe, R. (1982). A model for the teaching of games in secondary schools. Bulletin of Physical Education, 18(1), 5-8.

Chepyator-Thomson, J. R. y Ennis, C. D. (1997). Reproduction and resistance to the culture of feminity and masculinity in secondary school physical education. Research quarterly for exercise and sport, 68(1), 89-99.

Cockburn, C. (2000). Las opiniones de chicas de 13 y 14 años sobre la Educación Física en las escuelas públicas británicas. Estudio realizado en los condados de Hampshire y Cambridgeshire. Apunts: Educación Física y Deportes, 62, 91-101.

Columna, L., Arndt, K., Lieberman, L. y Yang, S. (2009). Using Online Videos for Disability Awareness. Journal of Physical Education, Recreation and Dance, 80(8), 19-24.

Connell, R. W. (1997). Escuelas y justicia social. Madrid: Morata.

Davis, R., Rocco-Dillon, S., Grenier, M., Martínez, D. y Aenchbacker, A. (2012). Implementing disability sports in the general physical education curriculum. Journal of Physical Education. Recreation and Dance, 83(5), 35-41.

Díaz, V. (2005). Manual de trabajo de campo en la encuesta. Madrid: Centros de investigación sociológica.

Eminovic, F., Nikic, R., Stojkovic, I. y Pacic, S. (2009). Attitudes toward inclusion of persons with disabilities in sport activities. Sport Science, 2(1), 72-77.

Gil-Madrona, P., Cachón-Zagalaz, J., Díaz-Suárez, A., Valdivia-Moral, P. y Zagalaz-Sánchez, M ${ }^{a}$ L. (2014). Las niñas también quieren jugar: la participación conjunta de niños y niñas en actividades físicas no organizadas en el contexto escolar. Movimento, 20(1), 103-124.

González, C. (2005). El deporte, una potencial herramienta formativa. Apunts: Educación física y deportes, 77, 97-101.

Grenier, M. y Kearns, C. (2012). The Benefits of Implementing Disability Sports in Physical Education: A Model for Success. Journal of Physical Education, Recreation and Dance, 83, 23-27.

Hill, G. M. y Cleven, B. (2006). AComparison of Students' Choices of 9th Grade physical Education Activities by Ethnicity. The High School Journal, 89(2), 16-24.

Hutzler, Y. (2003). Attitudes Toward the Participation of Individuals 
With Disabilities in Physical Activity: A Review. Quest, 55(4), 347-373.

Izquierdo-Gómez, R. y Díaz-Cueto, M. (en prensa). Joven con síndrome de Down y natación recreativa: posibilidades hacia la inclusión. Revista Internacional de Medicina y Ciencias de la Actividad Física yel Deporte.

Kyles, C. y Lounsbery, M. (2004). Project destiny: initiating physical activity for non-athletic girls through sport. Journal of physical education, recreation and dance, 75(1), 37-41.

Lavega, P., Planas, A. y Ruiz, P. (2014). Juegos cooperativos e inclusión en educación física. Revista Internacional de Medicina y Ciencias de la Actividad Física y el Deporte, 14(53), 37-51.

Liu, Y., Kudlácek, M. y Ondrej, J. (2010). The influence of paralympic school day on children's attitudes towards people with disabilities. Acta Universitatis Palackianae Olomucensis. Gymnica, 40(2), 6369.

Madrid, P. D., Prieto-Ayuso, A., Samalot-Rivera, A. y Gil, P. (2016). Evaluación de una propuesta extraescolar de conductas apropiadas en educación física y deportiva. Retos. Nuevas tendencias en Educación Física, Deporte y Recreación, 30, 36-42.

Martínez de Quel, Ó., Fernández, E. y Camacho, M. J. (2010). Percepción de dificultades para la práctica de actividad física en chicas adolescentes y su evolución con la edad. Apunts: Educación física y deportes, 99, 92-99.

Mitchell, S. A., Oslin, J. L. y Griffin, L. L. (1995). The effects of two instructional approaches on game performance. Pedagogy in Practice: Teaching and Coaching in Physical Education and Sports, 1(1), 36-48.

Mitchell, S. A., Oslin, J. L. y Griffin, L. L. (2006). Teaching sport concepts and skills ( $2^{\mathrm{a}} \mathrm{ed}$.). Champaign, IL. United States of America: Human Kinetics.

Molina, J. P. y Valenciano, J. (2010). Creencias y actitudes hacia un profesor de educación física en silla de ruedas: un estudio de caso. Revista de Psicología del Deporte, 19(1), 137-149.

Ocete, C., Pérez-Tejero, J. y Coterón, J. (2014). Propuesta de un programa de intervención educativa para facilitar la inclusión de alumnos con discapacidad en educación física. Retos. Nuevas tendencias en Educación Física, Deporte y Recreación, 27, 140-145.

Monge, M. y Monge, M. A. (2009). Cuerpos, mentes y aprendizajes diversos: la clase de educación física como modelo de una educación inclusiva en Costa Rica. Ágora para la EF yel Deporte, 9, 115-136.

Reina, R., López, V., Jiménez, M., García-Calvo, T. y Hutzler, Y. (2011). Effects of Awareness Interventions on Children's Attitudes toward Peers with a Visual Impairment. International Journal of

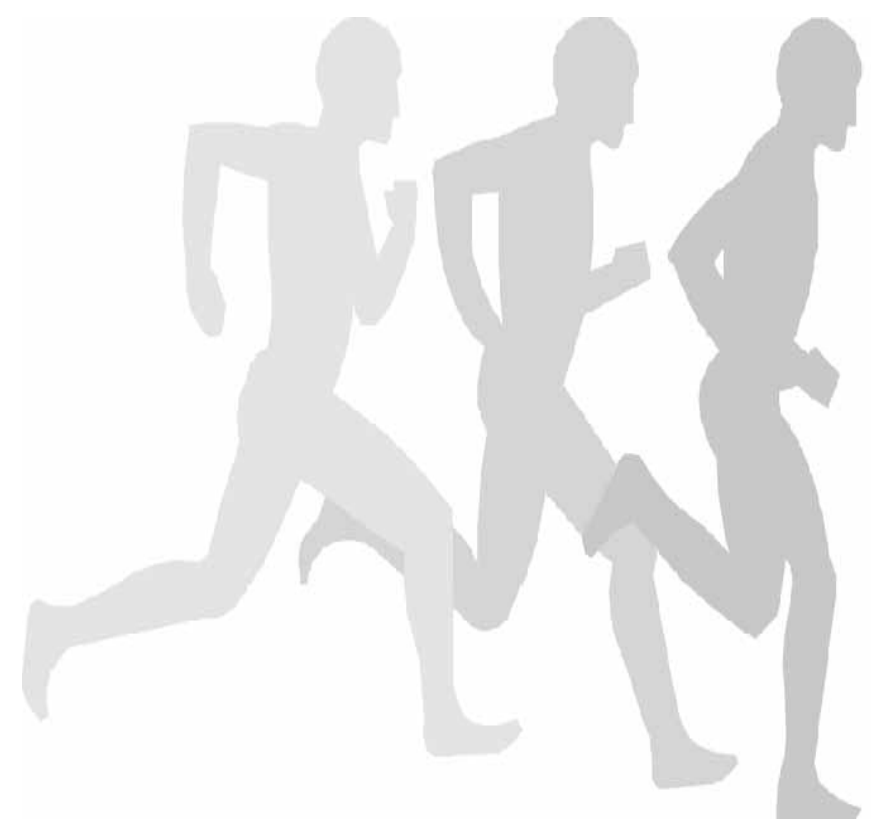

Rehabilitation Research,34(3), 243-248.

Robles, J. (2009a). Tratamiento del deporte Tratamiento del Deporte dentro del Área de Educación Física durante la Etapa de Educación Secundaria Obligatoria en la Provincia de Huelva. Tesis Doctoral: Universidad de Huelva.

Robles, J. (2009b). Tchoukball. Un deporte de equipo novedoso. Propuesta de aplicación en Secundaria. Retos. Nuevas tendencias en Educación Física, Deporte y Recreación, 16, 75-79.

Saiz, M., Prieto, A., Gutiérrez, E. C. y Gil, P. (2016). Evaluación inicial en $6^{\circ}$ curso de primaria de las habilidades sociales y propuesta didáctica de intervención en la Educación Física escolar. Sport TKRevista EuroAmericana de Ciencias del Deporte, 5(1), 64-75.

Santana, P. y Garoz, I. (2013). Actitudes hacia la discapacidad e intervención docente desde el deporte adaptado. Revista Internacional de Medicina y Ciencias de la Actividad Física y el Deporte, 13, 49, 1-17.

Sanz, D. y Reina, R. (2012). Actividades físicas y deportes adaptados para personas con discapacidad. Barcelona: Paidotribo.

Savino, L., Rio, L. y Gomez, F. (2015). The adapted physical activity as a valuable tool to overcome social prejudice to the disabled persons. Journal of Human Sport And Exercise, 10(1), 418-424.

Segura, J., Martínez-Ferrer, J-O., Guerra, M. y Barnet, S. (2013). Creencias sobre la inclusión social y el deporte adaptado de deportistas, técnicos y gestores de federaciones deportivas de deportes para personas con discapacidad. Revista Iberoamericana de Psicología del Ejercicio y el Deporte, 8(1), 127-152.

Seymour, H., Reid, G. y Bloom, G. A. (2009). Friendship in inclusive physical education. Adapted Physical Quaterly, 26, 201-219.

Smith, A. (2004). The inclussion of pupils with especial educational needs in secondary school physical education. Physical Education and Sport Pedagogy, 9(1), 37-54.

Spencer-Cavaliere, N. y Peers, D. (2011). «What's the difference? Women's wheelchair basketball, reverse integration, and the question(ing) of disability. Adapted Physical Activity Quarterly, 28, 291-309.

Turner, A. P. y Martinek, T. J. (1995). Teaching for understanding: a model for improving decision making during game play. Quest, (Champaign, III), 47(1), 44-63.

Ureña, N., Alarcón, F. y Ureña, F. (2009). La realidad de los deportes colectivos en la Enseñanza Secundaria. Cómo planifican e intervienen los profesores de Murcia. Retos. Nuevas tendencias en Educación Física, deporte y recreación, 16, 9-15.

Vaughn, B. (2012). Beyond Physical Education: Interscholastic Sports. Journal of Physical Education, Recreation and Dance, 83(3), 3233.

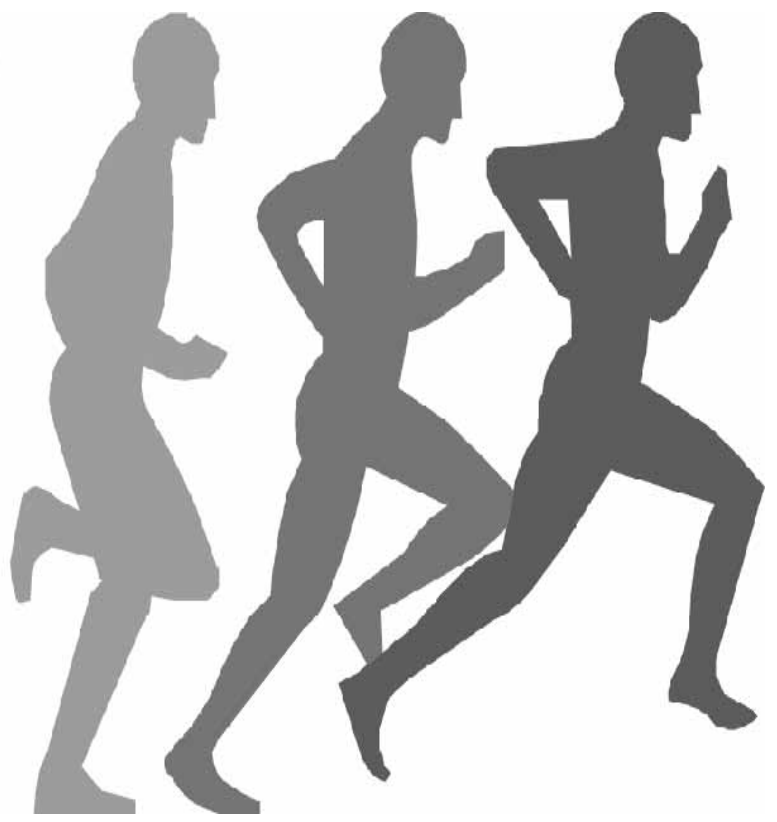

